\title{
EFL Learners' Grammatical Awareness through Accumulating Formulaic Sequences of Morphological Structure (-ing)
}

\author{
Kazuko Kashiwagi ${ }^{1} \&$ Yukiko Ito ${ }^{2}$ \\ ${ }^{1}$ Graduate School of Education, Osaka Kyoiku University, Osaka, Japan \\ ${ }^{2}$ Faculty of Management, Osaka Seikei University, Osaka, Japan \\ Correspondence: Kazuko Kashiwagi, Graduate School of Education, Osaka Kyoiku University, 4-88, \\ Minamikawahori, Tennoji-ku, Osaka, 543-0054, Japan. Tel: 81-6-6775-6636. E-mail: \\ kashiwag@cc.osaka-kyoiku.ac.jp
}

Received: September 2, 2017 Accepted: October 5, 2017 Online Published: October 8, 2017

doi: 10.5539/elt.v10n11p60 URL: http://doi.org/10.5539/elt.v10n11p60

\begin{abstract}
Even young EFL learners who have not yet learned L2 grammar will notice language patterns if, when retrieving exemplars ("item-based patterns"), they succeed in making form-meaning connections (FMCs). Item-based patterns, termed formulaic sequences (FS), serve as a basis for creative constructions. Although learners are implicitly sensitive to the frequency of the occurrence of constructions, item-based patterns are largely overlooked and are not retained. Because of the gap between elementary and secondary schools, students believe there is a difference between item-based patterns and the process of learning grammar. This phenomenon extends to EFL. The study investigated the extent to which Japanese students who had completed 150 hours of English lessons (age 13, $N=95$ ) noticed linguistic patterns when using a grammatical judgment test. Targeting the present progressive form -ing as FS, the teacher used three treatments: (a) recall of chunking, (b) structured input and dictogloss, and (c) a ten-minute inductive explication of grammar in L1. The results revealed significant differences between pre- and post-tests for awkwardness of word order $(31 \%<59 \%)$ and omission of morphemes: -ing $(61 \%<74 \%)$. Overall, students who had received the instructional medium exhibited grammatical sensitivity to FS.
\end{abstract}

Keywords: formulaic sequences, focus on form, grammaticality judgement tests

\section{Introduction}

In the previous study of Kashiwagi (2012), we investigated the extent to which young adolescent learners notice linguistic patterns when acquiring multiword units that are defined as formulaic sequences (FS). These fifth- and sixth-grade students (12 and 13 years old) learned English in a Japan's elementary school during the year 2012 in elementary school foreign language activities (known as "English activities") based exclusively on aural input. The lessons involved 1 hour of English activities each week (70 lessons in total) introduced in Japanese public elementary schools following new curriculum guidelines (Japan Ministory of Education, Culture, Sports, Science and Technology: MEXT, 2008). Examining the extent to which students in the same school area retained FS and identified linguistic patterns in spoken English, Kashiwagi (2012) found that, as well as noticing linguistic patterns, students detected the awkwardness of incorrect sentences.

In the successive previous study of Urata, Kashiwagi and Nakata (2014), we, more specifically, investigated to what extent adolescent learners (age 13) retained FS and whether FS would be transferred to English lessons at the next stage (first grade of junior high school; 4 hours per week during the first half-year). The study examined how existing FS effectively supported subsequent English learning; it was expected that FS would develop learners' sensitivity to linguistic patterns when distinguishing spoken sentences as grammatical or ungrammatical. The theoretical framework (elaborated in more detail later) is based on FS and the usage-based model ( $\left.\mathrm{UBM}^{1}\right)$, which has been improved by several linguists (Ellis, 2008; Ellis \& Larsen-Freeman, 2009; Logan, 1988; Nakamori, 2009; Skehan, 1998; Tomasello, 2003).

Prior to 2011, English education commonly began in the first year of junior high school; however, English lessons (designated as foreign language activities) have recently been introduced into elementary schools. English language teaching in Japan is at a turning point. All public elementary school students are expected to listen to English and use it 
more often in the classroom. At the end of sixth grade, students are likely to have acquired many exemplars through imitation and repetition in communicating with each other, even though they have not been taught grammar explicitly. These changes indicate that students now have a different language experience and can hear, imitate, repeat, and replace parts of chunks (e.g., draw a circle, draw a square) as needed, even before they enroll in junior high school.

In light of the above changes, it seems necessary to modify the teaching approach for first-year junior high school students. Under current teaching procedures, elementary school students learn new linguistic properties in a different way; whole prefabricated patterns are taught in a meaningful context and are then imitated and repeated by the students (e.g., "I can play baseball" or "Draw a circle on the whiteboard"); linguistic rules are not explicitly taught. However, the process by which students should access the meaning-bearing form during foreign-language activities has not been clearly stated. From the viewpoint of language learning, meaningful input is essential, and the students are encouraged to use chunks, replacing parts of sentences naturally in an interactive classroom ("I can play dodge ball" or "Draw a rectangle on the paper"). The italicized parts would be newly created by the teacher and students while engaging naturally in activities. These phenomena are superficially captured from the view point of junior high school English teachers, such as teachers saying, "the students' listening abilities have improved" or "The students seem to already know some words." On the other hand, English teachers who use an oral introduction in an interactive way from the outset are prone to report more precisely that "I only used a picture card to teach a word, morning, but the students reacted by saying in the morning" or "I only told the students to create a skit, self-introduction, in pairs, but the students added a simple expression such as "I live in X," "I don't like X," or "Go shopping." The students seem to be able to recall chunks in context. These phenomena seem to be grasped differently by English teachers who have not yet noticed the transition of FS and others who have already acknowledged it as linguistic properties the students come to stock. The latter linguistic phenomena are stated as form-meaning connections $\left(\mathrm{FMCs}^{2}\right.$ ). Vanpatten, William, and Rott (2004) suggested FMCs are the act of imitating and repeating; in particular, they embrace negotiation of meaning and connecting with linguistic patterns.

Although this procedure seems simple and feasible, it is significant for language development in FS. However, the importance of cognitive procedures and developmental mechanisms for later learning has not been clarified and is occasionally misunderstood by instructors. As a result, students' existing FS stock has been neglected, and often ignored, in English lessons at the beginning of junior high school. It is difficult for junior high school teachers to assess accumulated FS and FMCs established in elementary school; because this depends on aural inputs rather than written texts, it remains obscured. If the assumption that the current junior high school students (age 13) develop grammatical sensitivity through receiving the instructional medium using FS, then their process of FMCs and accessing linguistic patterns naturally by themselves should be illuminated and built upon in the next learning stage.

\section{Background of Study}

\subsection{Formulaic Sequences}

The present study was grounded in the concept of formulaic sequences, which originated in the study of mother-tongue acquisition. In recent years, cognitive linguists (Bybee, 2008; Goldberg, 1995; Langacker, 2000; Tomasello, 1998) have also advanced the usage-based model, which holds that abstract grammar emerges from language use (Langacker, 2000). Tomasello $(1992,2003)$ collected data from a diary of language utterances by his daughter Travis (T), who was between 2 to 3 years old. According to his entries about T's language development, the usage-based approach shows that natural language competence involves a much more complex and diverse set of linguistic representations than the "core grammar" of a formal approach (Tomasello, 2003, pp.5-6). Tomasello defined this "mastery of all its items and structures" as the item-based learning largely seen in children's early language development. Noting that children start to imitate item-based expressions, he referred to exemplars in terms of the "verb island hypothesis" as being isolated and mutually independent (e.g., get sauce, get me up there). Subsequently, children form a slot-filter category, such as (get $X)$. This cognitive procedure reflects human categorization and schema formation. Throughout the accumulation of item-based learning, category learning emerges, and children begin to analyze slots and generalize structures across isolated patterns. The process of verb acquisition is defined in terms of schema formation. The present study defines this cognitive procedure as FS. According to Yamaoka, the act of imitating and repeating does not mean "skill-based learning nor pattern practice but cognitive learning where children can develop FMCs" (Yamaoka, 2005, 2006).

The concept of applying the extensive research on FS to second language acquisition has attracted considerable interest. Skehan (1998) proposed that "learners must accumulate a repertoire of formulaic 'chunks' of verbs in order to become cognitively proficient" (p. 54). Logan (1988) claimed that fluent speech is based on the retrieval 
of ready-made exemplars (rather than on the rule), requiring only minimal processing capacity. In this sense, FS plays a dual role of cognitive dimension and rapid means of access to whole chunks. In the recent study, the importance of learning formulaic sequences was acknowledged (Ellis, 2008, 2012; Ellis \& Larsen-Freeman, 2009; Myles \& Cordier, 2017). Eskildsen (2012) reported that the frequency of FS English negation patterns among Spanish adult learners demonstrated an effect on English learning. Kidd, Lieven, and Tomasello (2010) found that children's (at age 6) repetition of the target sentence in L2 learning depended on the higher frequency input used by the teacher. However, both the quantity and quality of empirical research on FS in L2 classroom settings remains insufficient, especially regarding young adolescent learners.

To discover the effectiveness of exemplar-based learning in Japanese elementary school, Kashiwagi (2012) investigated the extent to which first- through sixth-grade Japanese children were able to establish FMCs of verb phrases (e.g., drink juice, change my clothes, give a flower to, and so on) while relying on degree of word familiarity. The students had been taught by aural input and received no grammar explanation. The level of FMCs increased noticeably in the fourth grade after 100 hours of English activities. In the same study, when ALTs demonstrated small talk about "a busy morning" using gestures and putting forth some questions, the record of student's imitative output showed that students who accumulated FS after 50 hours of lessons successfully picked up both VO and noun phrases. The increase in C-units ${ }^{3}$ of imitative output in the third grade confirmed that these children had started to categorize input and seemed to segment the salient prefabricated patterns.

In terms of language learning, it has also been reported that FS has a positive impact on the development of procedural knowledge ${ }^{4}$ (compared to declarative knowledge ${ }^{5}$ ), providing a foundation for the practical use of English. Gass (1997) mentioned that the frequency of a particular target structure can impact the "noticing" forms. The more learners accumulate FS, the more noticing occurs. Bybee (2008) suggested that procedural knowledge is "categorization" and analogy-forming and is crucial in determining the production of constructions. Both studies showed that the ability to construct categories and analogies, indicating procedural knowledge, can be enhanced when learners accumulate a range of FS. Additionally, FS serves as a language slot (e.g., get $X$, draw $X$ on the $Y$ ) until beginning learners catch up with grammar rules taught by rote learning. As Myles (2004) claimed:

L2 uttered by the 16 beginners who learn English and French at the age of 7-9 and 9-11 in the classroom where they utilize formulaic sequences regarding 'multimorphemic sequences'...chunks do not become discarded: they remain grammatically advanced until the grammar catches up, and it is this process of resolving the tension between grammatically advanced chunks and the current grammar which drives the learning process forward. ( $\mathrm{p}$. 152)

Therefore, it can be hypothesized that the 13-year-old students participating in the present study would demonstrate their grammatical sensitivity in terms of adhering to linguistic features, awkwardness of incorrect word order, and so on. On this assumption, learners would be better able to pay attention to input as linguistic property. As Skehan (1998) stated about dual mode, ${ }^{6}$ if learners' procedural knowledge can be raised, even fast learners (and adult learners) will be able to learn a new language from both explicit grammar rules and analogical inference from the input (both aural and written). This would bring lasting benefits to Japanese language learners, who could easily access whole chunks rapidly.

\subsection{Instance to Rule}

The present study investigates students' ability to find linguistic patterns in FS based on cognitive processing of "instance to rule" rather than "rule to instance." Anderson (1993) defined adaptive control of thought-rational (ACT-R) as something in which "production rules are created" by learners "initially by finding and experiencing the connection between antecedents and consequences in individual exemplars as instances of these connections, and then by generalizing them through repeated experiences with further exemplars" (p. 293). Anderson's experiment involved eight examples, and participants practiced application of the rules to these examples over a period of 4 to 5 days. Anderson, Fincham, and Douglass (1997) found that participants began from pure exemplar-based processing, in which they acquired procedural skills by retrieving examples. Here, exemplar-based learning (FS) at the initial stage is a fundamental language experience that may develop procedural skills and help learners create rules at the later stage. Yamaoka (2008) discussed the importance of the "instance to rule" stated in ACT-R and how the concept might be realized in elementary school English activities, suggesting that "teachers should encourage students not only to repeat simple everyday phrases but also to learn and be able to utilize more practical expressions that represent the realization of teachers themselves, so students can transition from thinking concretely to being able to think abstractly."(p.83). For students who are 12 to 13 
years old (the formal-operational stage), Menyuk and Brisk (2005) also pointed out that abstraction (instance to rule) in the students" "mental operation" would be applicable to any and all conceivable content. For instance, formal thinking at this age distinguishes between reasoning about what is possible in all possible worlds and what is possible in the actual world. In the current study, it is proposed that FS and compatible linguistic information should be used when teachers share their innermost thoughts or feelings with students in the classroom.

However, if the FS the students' (age 12) stock is not taken over to the next stage (age 13), where they would start being instructed by explaining grammar rules in their mother language, remembering words by breaking them down and then practicing the mechanical drills, there will be no route to follow regarding finding linguistic patterns and being interested in a new language's property.

\subsection{The Previous Study}

There is a scarcity of empirical classroom research regarding the adolescent age. Therefore, to clarify students' established FMCs and their grammatical sensitivity to linguistic patterns, Urata et al. (2014) conducted research to the extent that students notice linguistic patterns; here, the sound-grammaticality judgement test (S-GJTs) was used. A higher score indicated grammatical sensitivities because of the accumulation of FS. Participants included 41 elementary school students (group A, mean age: 12.5) and 40 junior high school students (group B, mean age: 13.5).

Group A students were tested after approximately 50 hours of English lessons (taught once a week for a year and a half). S-GJTs were conducted; the results (as reported by Urata et al, 2014) are presented in Table 1. Based on a test that was sound recorded by a native speaker and read twice at intervals of 2 seconds, students were required to find linguistic patterns, such as correct word order ( $73 \%$ to $98 \%$ correct answers); ungrammatical sentences, such as incorrect word order negation (87.8\%); insertion between $V$ and $O(85.4 \%)$; incorrect VO (75.6\%); and omission-verb (58.2\%). The results indicated that these students could judge both sentence correctness and the awkwardness of ungrammatical sentences, and they retained FS (Table 1).

When tested, the junior high school students in group B had completed approximately 80 hours of English lessons. The students had previously experienced English activities for 2 years in elementary school. On enrolling in junior high school, they learned English with four skills and learned basic English grammar skills: copula -be, present form, present progressive form, and third person singular -s, as well as nouns, adjectives adverbs, and VO, (but not can and VO). As for group A, the same S-GJTs test was conducted prior to the lesson titled "the use of auxiliary 'can", (Kairyudo, 2014). The results of the S-GJTs are presented in Table 1. Students in this group were again asked to identify linguistic patterns, judging the grammaticality of sentences in terms of correct word order (77\% to $98 \%$ correct) and ungrammatical items such as incorrect word order negation (85.2 \%), insertion between $V$ and $O(86.1 \%)$, and omission-verb (56.1\%). In general, group B's scores were similar to or slightly better than group A's scores. Surprisingly, although the score for omission-the increased, the score for incorrect $V O$ declined (21.5\%).

Both groups of students could find linguistic patterns and performed well in their sense of sentence correctness and awkwardness (for incorrect word order, insertion, and incorrect VO although not for omission). Overall, group A students achieved good scores, even though they had learned English only aurally, without being taught grammar explicitly. Group B students showed good retention of FS but showed no significant difference in S-GJT scores. For instance, the score for "I can play swim" was lower for group B (21.5\%) than for group A (56.1\%). This indicates that the elementary school students were stronger in detecting incorrect prefabricated patterns, such as "I can play swim" and that they may retain FS as the continuity between words. The results indicated that chunks, FS as cognitive procedures, and their use in the interactive classroom should not be discarded. 
Table 1. S-GJT Scores for Can + VO (adapted from Urata et al., 2014)

\begin{tabular}{|c|c|c|c|c|}
\hline \multirow[t]{2}{*}{ No. } & \multirow[t]{2}{*}{$\begin{array}{l}\text { S-GJTs } \\
\end{array}$} & \multirow{2}{*}{$\begin{array}{l}\text { correct or incorrect } \\
(\mathrm{VO} \text { phrases })(\text { can }+\mathrm{VO})\end{array}$} & \multicolumn{2}{|c|}{ Correct answers $(\%)$} \\
\hline & & & Age 12.5 & Age 13.5 \\
\hline 1 & I can cook. & $x$ & 97.6 & 98 \\
\hline 2 & I can soccer. & $\checkmark \quad$ Omission-Verb & 56.1 & 58.2 \\
\hline 3 & I can play swim. & $\checkmark \quad$ Incorrect $V O$ & 75.6 & 21.5 \\
\hline 4 & I can play table tennis. & $x$ & 92.7 & 95.4 \\
\hline 5 & I play can Kendama. & $\checkmark \quad$ Insertion between $V \& O$ & 85.4 & 86.1 \\
\hline 6 & I can play piano. & $\checkmark$ Omission-the & 26.8 & 56.3 \\
\hline 7 & I can't play the recorder. & $x$ & 73.2 & 77.2 \\
\hline 8 & I play can't basketball. & $\checkmark \quad$ Incorrect word order-negation & 87.8 & 85.2 \\
\hline
\end{tabular}

Note. Italic: Japanese words; $V$ : verb; O: object

$\boldsymbol{X}$ :correct sentences $\mathbb{\checkmark}$ :incorrect sentences

Age 12.5: Elementary school students; Age 13.5: Junior high school students.

Subsequently, to address the next learning stage, the present study targeted FS (grammar structures $V O$ in the present progressive and collocation of foregoing and subsequent VO). This included the morphological structure -ing that students frequently hear in storytelling or in a teacher's oral introduction.

\subsection{Overview of Teaching English Grammar in Japan}

Based on this understanding of FS and ACT-R, we reviewed current grammar teaching in Japan and considered the applicability of "instance to rule." Regarding junior high school English education, MEXT (2008) stated that one overall objective is to develop basic communication abilities. MEXT (2017) announced that teaching grammar must be fully integrated with meaningful language activities rather than depending on grammar translation and that linguistic rules should be taught to support communication. As a traditional approach to teaching language based on explicit instruction, the use of grammar translation in both EFL and ESL classrooms has been criticized on the grounds that grammatical objects are taught without reference to any meaningful context. The instructional approach may "largely ignore language learning process" (Long \& Robinson, 1998, p. 16). It also produces more false beginners than finishers because it ignores beginners' own possibilities (human capacity for language acquisition to be highly resilient; Long, 1997). In contrast, four approaches to teaching grammar in support of communication are as follows.

- Focus on form (FonF) is an instructional process that prompts children to identify linguistic features by providing meaningful input (Doughty \& Williams, 1998; Gass, 1997; Izumi, 2009; Long \& Robinson, 1998; Muranoi, 2006; Shintani, 2012, 2013). Schmidt (1995) claimed that language acquisition does not occur without noticing linguistic features, and FonF allows students to find language patterns by repeatedly providing exemplars and interchanging words within slots. Specifically, this process can be realized by using token frequency ${ }^{7}$ combined with type frequency ${ }^{8}$ (e.g., draw a circle on it can be exchanged with draw X on Y). To direct students' attention to the form, teachers may use structured input, input enhancement, recast, dictogloss, ${ }^{9}$ consciousness-raising tasks, input processing, garden path, and others (Doughty \& Williams, 1998, p. 258).

- Presentation-practice-production (PPP) has been employed in both ESL and EFL classrooms. This approach is more deductive than FonF, emphasizing earlier discussion of grammar rules using an oral introduction; it does not wait for learners to notice linguistic patterns. PPP aims to develop declarative knowledge (Skehan, 1998) and focuses on accuracy in production. Sato (2011) suggested that Japanese learners with little exposure to English would notice fewer linguistic patterns than with FonF and that some version of PPP is more suitable for teaching Japanese students.

- As a better alternative, depending on the difficulty of targeted grammar, some SLA researchers (Dulay \& Burt, 1974; Izumi \& Isahara, 2004; Shirahata, 1988; Shirahata, Suda, \& Wakabayashi, 2006) have suggested using natural acquisition order, which is influenced by the learner's mother language. For instance, the morphosyntactic structure -ing (similar to -teiru morphemes in Japanese) ranks second while the article "the" 
(which does not exist in Japanese) ranks last because of the learner's L1 influence. In this case, it is considered that the former grammar can be taught with FonF in which procedural knowledge could be developed and the learners may notice language patterns while the latter one could be taught with PPP in which declarative knowledge will support an understanding of the rules.

- Finally, the present study proposes FS. In one of the very few empirical studies of FS in Japan, Nakamori (2009) claimed that this approach is suitable for junior high school students because "It does not explain grammar rules in an abstract manner and children can understand what is going on. Children can recognize how lexis and chunks work in a natural setting." Additionally, Nakamori (2009) stated, "Human cognitive development requires that explicit grammar instruction should be given if and only if the learner has matured enough to process the abstract rules" (p. 161).

The present study promotes this last option for young adolescent learners (ages 12 and 13), taking the FS that the students come to possess into consideration. In addition, the instructional medium of FonF is also essential. Using an explicit grammar explanation in an inductive way is also heightened by eliciting the students' noticing the patterns. The third option might be considered effective because the targeted grammar in the present study is "morphosyntactic structure-ing," which is ranked as an L1 positive influence. For the most part, we take into account the "instance to rule," which improves procedural knowledge. The instructive ways are (a) to provide exemplars as linguistic property (FS-exemplars) using teacher talk; (b) to elicit FS accumulated by students prior to the lesson, providing an opportunity to access the rule on the basis of their own FS-accumulation; (c) to provide an explicit grammar explanation using the chart after students have formed a schema of the structure (FS-schema formation); and (d) to encourage students to imitate and repeat, borrowing the exemplars for production (FS-borrowing). With the exception of (c), all these procedures should be taught in English. The teacher should encourage students to replace the FS part by borrowing the given exemplars rather than creating new sentences. FS exemplars written on teachers' handouts can also be borrowed. For instance, between (b) and (c), the teacher needs students to find linguistic patterns using dictogloss ${ }^{9}$ (Wajnryb, 1990) with sound and structured input ${ }^{10}$ (FS-FonF). The grammar image acquired by the student is drawn on the chart (fewer grammar terms means less tedious explanations). In (d), the teacher must ensure that students do not rely on the rule but that they instead use "imitating," "repeating," "borrowing," and "replacing parts of chunks" (increasing both token and type frequency) to form language slots that students can access rapidly). In (d), the grammar rule learned in (c) can be used by students to express their own ideas.

\section{Purpose of the Study}

In general, the study aimed to investigate the effectiveness of FS in a classroom setting. The research objectives were as follows:

1) To develop and conduct an English class based on FS, activating students' FS that they come to possess using sound (imitating, repeating, and replacing parts of the language slot), encouraging students to establish form-meaning connections (FMCs) and notice linguistic patterns.

2) To investigate the extent to which students pay attention to these patterns (here, the morphosyntactic structure -ing) using pre- and post sound grammaticality judgement tests (S-GJTs); to assess students' grammatical sensitivity to each item (including linguistic patterns such as morphemes and word order); and to examine how each item changes during experimental practice between the pre- and post-test.

3) To consider whether FS and detection of linguistic patterns impacts positively on students' language learning and their development of procedural knowledge.

\section{Methodology}

\subsection{Participants}

The participants were first-grade junior high school students (Mean Age $=13.7 ; N=95$ ). Their English proficiencies, as measured by self-recognized EIKEN grade levels, were Grade 5, with roughly $60 \%$ of students within Grade 5 and 30\% within Grade 4. A J-JET (Y) taught the English lessons in all these classes using the same FS procedures ( 8 hours in total). Prior to the present study, the students had experienced English activities for 2 years (twice a week in fifth and sixth grade) when they were in elementary school. Students began learning phonics in the fifth grade (age 11), paying attention to the initial sound and phonemes within the word by learning the concept of onset and rhyme (e.g., f・ox, $c \cdot a t)$.

However, they were not yet at the decoding stage when they enrolled in junior high school. They continued to learn decoding by listening to stories from more than 20 books in the Oxford Reading Tree; the book we used was from this collection (Hunt, 2003), and the J-JET (Y) posed some questions about the story in an interactive 
style. J-JET (Y) taught all the English lessons for participants. The lessons were taught in English, and the flow of the lesson was "rule to instance." The grammar items that students had already learned before the experimental practice were copula-be, verbs, third person singular-s, and auxiliary can. The present study did not employ a comparison or control group; instead, a one-group, pre- and post-test design was used, taking into account the students' introductory learning stage.

\subsection{Lesson Procedures}

The lesson was taught using "instance to rule," which differed from the existing approach. Conducted in January in the first grade of junior high school, the total duration of the lessons was 8 hours.

Prior to the first lesson, the pre-test, timed grammaticality judgement tests, was conducted.

First lesson: FS (exemplar-based learning)

Following the FS procedure, the teacher first elicited existing FS (present progressive -ing) interactively by showing picture cards: Hi, Friends! 2: "What is $\mathrm{X} \sim$ ing?" "X is $\sim$ ing." The lesson used verb phrases that students had already learned (e.g., swim, play soccer, study math, cook dinner).

Second and third lessons: FS (FMCs, FonF, accumulation, schema formation)

The teacher introduced storytelling to draw the students' attention to the present progressive -ing in the context, using only sound and asking questions using gestures. The students were then challenged to retell the story in groups (dictogloss from the sound called S-dictogloss in the study).

Fourth and fifth lessons: FS (teaching grammar in an inductive way)

The students opened their textbooks and listened to the content before reading the text and practicing exercises orally using the present progressive -ing. The teacher then explained the grammar explicitly, using the chart and asking the students in Japanese (for 10 minutes) what sentence patterns they noticed (1: be $+\sim$ ing; 2 : Are you $\sim$ ing? 3: What are you $\sim$ ing?). Inverted $V O$ and -ing after a verb (e.g., changing clothes now) was also taught using VO phrases.

Sixth, seventh, and eighth lessons: FS (imitating, repeating, borrowing FS, token, and type frequency)

The teacher again used storytelling and S-dictogloss before asking the students to create a skit in groups. The production replaced the FS part and added some sentences rather than creating a new story.

Then, the post-test, timed grammaticality judgement tests, was conducted.

The examples used in the story and the model sentences used in the textbook are shown in Appendix 1 and Appendix 2, respectively. For instance, the students encountered exemplars of the present progressive, such as "A magic key is glowing now" more than six times through S-dictogloss, storytelling, and interactions between the teacher and students. To draw the students' attention to the sound form, storytelling did not include any alphabet letters or pictures.

\subsection{The Data Analysis Procedure}

To evaluate procedural knowledge, timed grammaticality judgement tests: $S$-GJTs were performed. In this test, the examinees had to judge intuitively whether a sentence was grammatically correct or incorrect in a limited time. S-GJTs measure three main processing operations: (a) semantic processing, (b) noticing, and (c) reflecting. Although (a) and (b) reflect the learner's procedural knowledge, (c) reflects one's declarative knowledge (Loewen, 2009, Ellis, 2005). Investigating what kind of knowledge was involved in judgements of correctness and incorrectness of GJTs, Xavier (2013) reported, "The learners judged grammatical sentences significantly more accurately than ungrammatical ones in both timed and the untimed GJTs. This ability might be due to the learners' implicit knowledge. Discriminating ungrammatical sentences might be due to the learners' explicit knowledge" (p. 442).

The tests employed sound recorded by a native speaker; to observe participants' grammatical sensitivity through learning FS using mainly input and interaction, written sentences were not shown. We characterized these tests as S-GJTs. In the test, the first example was used to practice and demonstrate the test procedure. Each test sentence was repeated twice at intervals of 2 seconds. The tests consisted of 10 items (10 points in total), including omission-copula-be, incorrect insertion, subject-verb (SV) disagreement, incorrect word order and inverted VO, and incorrect insertion between Vi and preposition (see Tables 2 and 3). Scores were compared between the pre-test and post-test. For the pre-test, we used verb phrases that were familiar to participants from English activities (e.g., I am listening to music). For the post-test, we used verb phrases (e.g., Are you cleaning your own room now?) in the storytelling and textbook stories the participants listened to (see Appendix 1 and 
Appendix 2).

\subsection{Reliability and Validity of S-GJTs}

Although there is no preceding study of S-GJTs conducted with sound, some previous studies have used text-based GJTs, including Ellis (2005) and Shimada (2014), who sought to measure explicit and implicit knowledge. In the present study, the reliability of the post-test was measured using a repeated test (post-test $\alpha$ ) for the same participants a year later. If the post-test itself and post-testa showed a positive correlation, the test's reliability would be confirmed. The validity of the post-test was also measured using post-test $\beta$, a listening proficiency test addressing the participants' FS and detection of linguistic patterns in parallel. We observed participants' time-restricted grammatical judgment, requiring them to pay attention both to meaning and form. Post-test $\beta$ contained (a) agreement of a story with the illustration; (b) selection of a correct answer to a question about a long skit; and (c) agreement of a question in a speech with the speech context. Post-test $\beta$ was conducted a year later; if both post-test and post-test $\beta$ showed a positive correlation, the test's validity would be confirmed. We examined the correlation between pre-test and post-test to assess whether the two tests measured the same grammatical judgement ability, confirming an indirect connection with post-test $\beta$ as well.

The post-test and post-test $\alpha$ revealed a positive correlation (post-test, $N=95$, Mean $=6.97, S D=1.77$; post-test $\alpha$, $N=95$, Mean $=7.82, S D=1.90$ ). Between the tests, it was shown that $N=95 ; r=.55 ; p<.01$; the reliability of the post-test was proved. Both the post-test and post-test $\beta$ also revealed a positive correlation (post-test, $N=95$, Mean $=6.97, S D=1.77$; post-test $\beta, N=95$, Mean $=82.3, S D=20.3$, Max $=100$, Min $=34$ ). Between the tests, the results were $N=95 ; r=.63 ; p<.01$; the validity of the post-test was proved. The pre-test and post-test revealed a positive correlation (pre-test, $N=95$, Mean $=5.9, S D=1.63$; post-testa, $N=95$, Mean $=6.97, S D=$ 1.77. Between the tests, $N=95, r=.46 ; p<.01$ ). Both the pre-test and post-test can be considered as validly measuring the same grammatical judgement ability. Posterior to the post-test, the class had been taught by the same J-JET(Y) and FS because instructional medium was mostly used after the current study. Between the post-test and post-test $\beta$, the students learned a past tense, a future tense, an infinitive, and a comparison through the same procedure "instance to rule." Present progressive had not been taught specifically once again; however, they had seen the morphological structure -ing in their production when they created the skit. The storytelling the teacher instructed often embraced it naturally. Considering the students who are at the introductory period, the number of test items needed to be limited. Therefore, 10 items in each test consisted of a variety of grammar items, such as omission, insertion, and word orders. On this account, the tests did not presume consistency, and the Cronbach $\alpha$ of each test was not relatively high (pre-test, $\alpha=.44$, post-test, $\alpha=.60$, post-test $\alpha=.63$ ).

\section{Results}

\subsection{Reporting on the Practice}

In the practice round, the teacher first performed storytelling using only sound. The students paid close attention to the story, even though the teacher told it without showing the illustration. They recalled the story in groups and reconstructed it, taking notes and writing down the fragments they recalled (Figure 1). Their notes revealed the extent to which they used chunks, such as copula-be, verbs, adverbs, and prepositions. Words were not isolated but were collocated instead. The students' collaborative learning while reconstructing the story in groups is shown in Figure 2. It became clear that although no written present progressive -ing appeared in their notes, they imitated the chunks with or without -ing when the teacher elicited orally what they had retained. In S-dictogloss, the participants had connected FS and its meaning, and they came to understand what the present-progressive -ing meant in the context of the story's plot, where "the hero is approaching and continuing" or "a new thing is happening now and ongoing here." The approach provided many chances for students to establish FMCs and to acquire a variety of exemplars (token frequency), especially for the present progressive -ing.

The students may have felt that the grammar (-ing) had an important function for relating their thoughts rather than as written grammar knowledge on paper. In the story, the students burst into laughter because of a funny mismatch between their image of the story and the story shown with the illustration - for example, the scene entitled, "The witch is thrown away" was shown only in the illustration. Then, the teacher gave the students an explicit grammar explanation in only 10 minutes. Before this, the students had already grasped when -ing is used and how it should be placed after verbs. The teacher did not need long for the explanation, and the students moved on to the practice and production activities. Some students told the teacher, "We understood the grammatical structure firmly in this way." Finally, the teacher asked the students to make a skit using the story's plot or the textbook's script. They seemed to already know many FS and replaced parts of the FS they knew, adding in some terms they already knew to areas that were new. Understanding grammar through cognitive 
procedures may play a significant role in language acquisition.

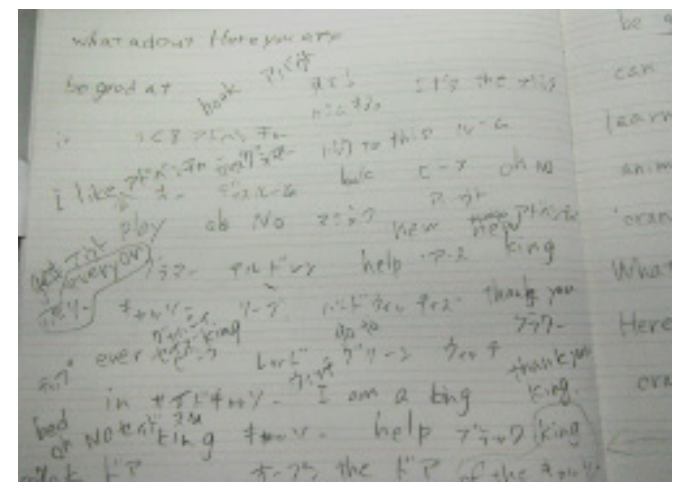

Figure 1. Notes reconstructed through S-dictogloss (in groups).

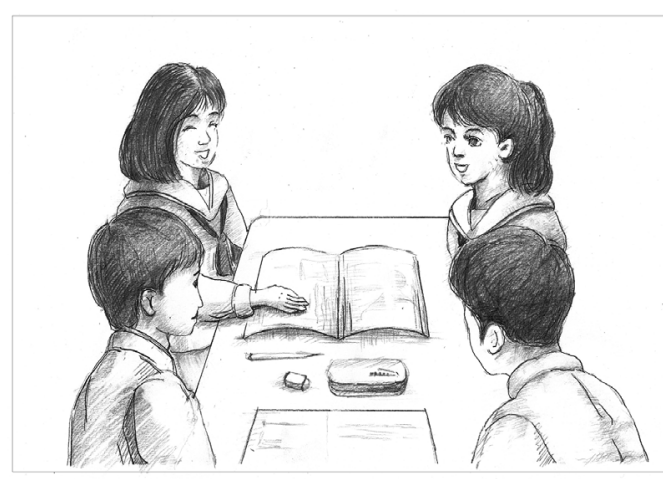

Figure 2. Recalling the story through S-dictogloss (in groups).

\subsection{Analysis of the S-GJT Results}

The pre-test and post-test results for each test item (percentage of correct answers displayed as a decimal value) are shown in Tables 2 and 3, respectively; the scores for each item are represented graphically in Figure 3. Total score, descriptive statistics, and the $t$-values for the repeated tests are shown in Table 4 . The change from pre-test to post-test is presented as a box plot graph in Figure 4. Pre-test and post-test data are displayed as a histogram to visualize the classroom distribution (Figures 5 and 6).

Summarizing the results of the pre-test for correct word order, participants returned approximately $80 \%$ correct answers (No. 1: 0.85 ; No. 2: 0.81; No. 3: 0.64). For omission -ing, participants returned approximately $60 \%$ correct answers (No. 4: 0.66; No. 5: 0.56). Participants readily grasped linguistic patterns such as "Are you X -ing?" and "I am X -ing." Regarding incorrect word order, incorrect insertion between Vi and preposition, and inverted $V O$, participants achieved lower scores, less than 50\% (No. 9: 0.31; No. 10: 0.46).

Table 2. Pre-test: S-GJTs

\begin{tabular}{|c|c|c|c|}
\hline No. & Pre-test: S-GJTs & Target grammatical item & $\%(d)$ \\
\hline 1 & Mr. $Y$ and Ms. $I$ are teaching English now. & $x$ & 0.85 \\
\hline 2 & I am making sushi now. & $x$ & 0.81 \\
\hline 3 & They are studying math now. & $x$ & 0.64 \\
\hline 4 & Are you clean your room now? & $\checkmark$ Omission -ing & 0.66 \\
\hline 5 & I am listen to music now. & $\checkmark$ Omission -ing & 0.56 \\
\hline 6 & Ken is playing skate now. & $\checkmark$ Insertion after $\mathrm{Vi}$ & 0.56 \\
\hline 7 & She helping your friend now. & $\checkmark$ Omission copula-be & 0.69 \\
\hline 8 & $Y$ and $T$ is cooking dinner now. & $\checkmark \mathrm{SV}$ disagreement & 0.41 \\
\hline 9 & I am coming school to now. & $\checkmark$ Insertion between $\mathrm{Vi}$ and preposition & 0.31 \\
\hline 10 & He is the door painting now. & $\checkmark$ Inverted VO & 0.46 \\
\hline
\end{tabular}

Note: The subject in Nos. 1 and 8 is a proper noun written as an initial.

Italics in S-GJT indicate errors.

$\%(d):$ Percentage of correct answers displayed as decimal values. 
Table 3. Post-test: S-GJTs

\begin{tabular}{llll}
\hline No. & Post-test: S-GJTs & Target grammatical item & $\%(d)$ \\
\hline 1 & A red witch is coming now. & $x$ & 0.70 \\
2 & I am cooking breakfast now. & $x$ & 0.87 \\
3 & A magic key is glowing now. & $x$ & 0.73 \\
4 & Are you dry your hair now? & $\checkmark$ Omission -ing & 0.67 \\
5 & I am change my clothes now. & $\checkmark$ Omission -ing & 0.79 \\
6 & Mr. $Y$ is playing ski now. & $\checkmark$ Insertion after Vi & 0.78 \\
7 & She using her computer now. & $\checkmark$ Omission copula-be & 0.69 \\
8 & B and K is jumping on the bed now. & $\checkmark$ Insertion between Vi and preposition & 0.63 \\
9 & They are swimming the pool in now. & $\checkmark$ Inverted VO & 0.59 \\
10 & Grandpa is ozoni making for us now. & & 0.51 \\
\hline
\end{tabular}

Note. The subject in Nos. 6 and 8 is a proper noun written as an initial.

Italics in S-GJT indicate errors.

$\%(d)$ : Percentage of correct answers displayed as decimal values.

However, regarding omission copula-be, participants scored relatively higher at above 50\% (No. 7: 0.69). Although it seems to have proved difficult for these students to judge omission, they were familiar with FS such as "she is," where the is was omitted, and they sensed this awkwardness. Regarding SV disagreement (No.8: 0.41), participants scored lower; this was not judged in the pre-test. For incorrect insertion after Vi, participants achieved a reasonable score of approximately 50\% (No. 6: 0.56).

Overall, the ability to judge correctness was higher, and participants could identify awkwardness when part of the FS was omitted. However, their ability to judge incorrectness of word order or SV disagreement was lower. Even though participants had not been taught about the present progressive -ing, they could detect the structure and sufficiently judge its correctness. The learning experience of establishing FMCs and "accumulation a repertoire of FS" with sound can be said to provide a foundation and is a predictor for the next learning stage. Although it is impossible to compare students who learned English (four skills at the same time) a decade ago to current ones, the current students' accumulation of FS and their "cognitive proficiency" may differ.

Turning to the results of the post-test, participants achieved $70 \%$ to $80 \%$ correct answers for correct word order (No. 1: 0.7 ; No. 2: 0.87 ; No. 3: 0.73 ). Regarding omission -ing, participants again returned approximately $70 \%$ to 80\% correct answers (No. 4: 0.67; No. 5: 0.79). Participants readily grasped linguistic patterns such as "Are you X -ing?" and "I am X -ing." Regarding incorrect word order: incorrect insertion between Vi and preposition and inverted $\mathrm{VO}$, the participants scored better than in the pre-test although this score remained at around $50 \%$ (No. 9: 0.59; No. 10: 0.51). More than half of the participants predicted what would come after a verb (cf. ozoni making and making ozoni) and felt the awkwardness of the pool after swimming (cf. swimming the pool in and swimming in the pool). An explicit grammar explanation may clarify that "swim" is an intransitive verb and does not connect with an object. However, the students' accumulated FS enabled them to judge the incorrectness of what comes after the verb "swim" using minimum cognitive processing operations rather than relying on the rules. Regarding omission-copula-be and SV disagreement, the participants scored higher (No. 7: 0.69; No. 8: 0.63). For incorrect insertion after Vi, participants scored much higher (No. 6: 0.78). Overall, the ability to judge correct word order, omission, and insertion proved to be higher, and they were able to detect awkwardness when part of the FS was missing or when the whole FS was divided unreasonably. Because the present progressive -ing and SV agreement were explicitly taught for 10 minutes in the lesson, these items may have been positively influenced by explicit knowledge of grammar. For incorrect word order, incorrect insertion between Vi and preposition, and inverted $\mathrm{VO}$, the scores were higher, indicating that these items had been positively influenced by FS.

On the whole, as seen in Table 4 and in the difference between the pre- and post-test in Figure 3, the overall score improved significantly from pre-test to post-test (pre-test: $d f=94$, Mean $=5.9, S D=1.63$; post-test: $d f=$ 94, Mean $=6.97, S D=1.765 ; t=5.873, p=.000, r=.52$, large effect size). It can be inferred that FS had a positive influence on the ability to judge both correctness and incorrectness in the limited available time, and 
there was greater retention and demonstration of grammatical sensitivity to linguistic patterns.

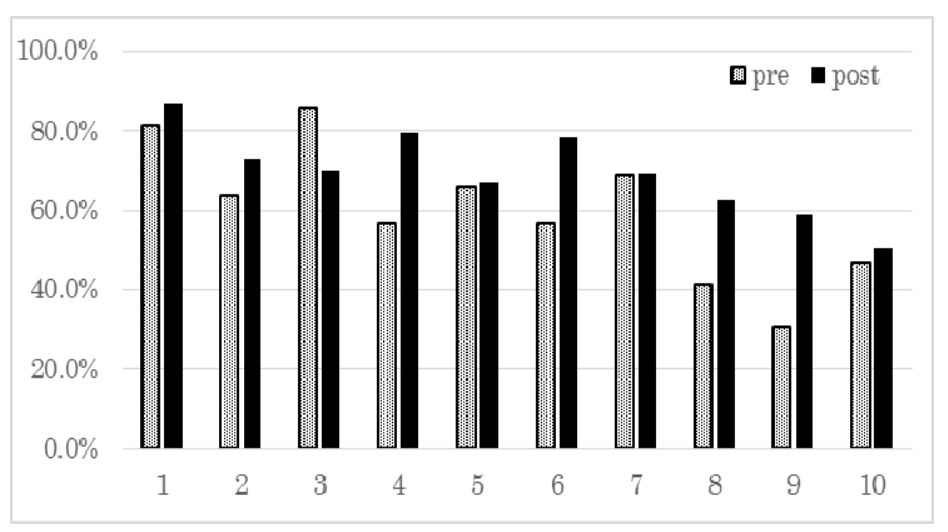

Figure 3. Comparison of post-test with pre-test $(N=95)$

Note. Horizontal axis: Number of the test items;

Vertical axis: The percentage of correct answers.

Table 4. Descriptive Statistics Comparing Pre-test and Post-test $(\mathrm{N}=95)$

\begin{tabular}{lllllllll}
\hline & $d f$ & Ave. & $S D$ & Min. & Max. & t-value & $p$-value & Effect Size $r$ \\
\hline Pre-test & 94 & 5.9 & 1.63 & 2 & 9 & 5.873 & .000 & 0.52 (Large) \\
Post-test & 94 & 6.97 & 1.765 & 3 & 10 & & & \\
\hline
\end{tabular}

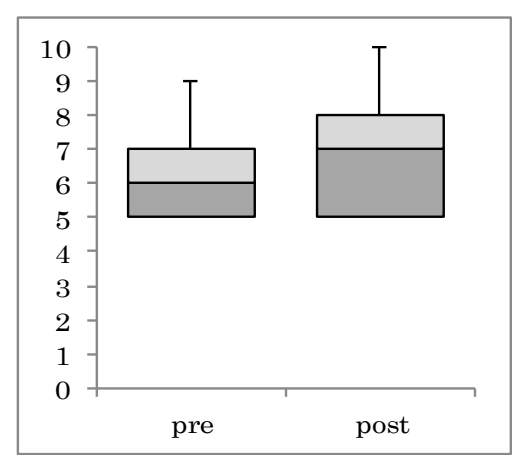

Figure 4. Graphic comparison of post-test with pre-test

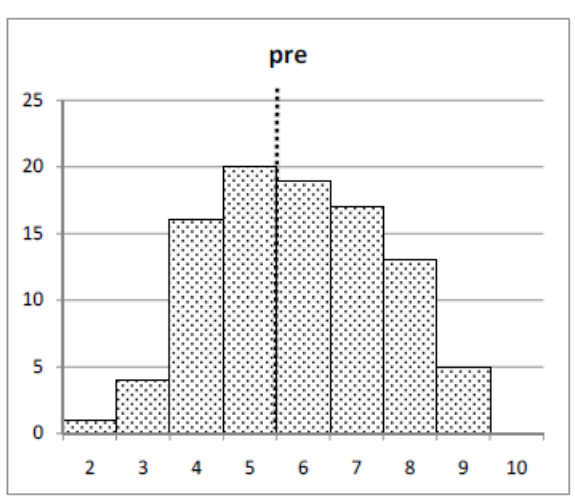

Figure 5. Histogram, pre-test $(N=95)$

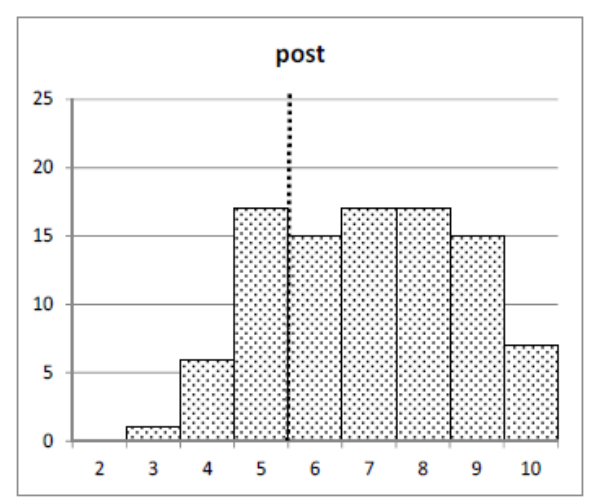

Figure 6. Histogram, post-test $(N=95)$ 
Figure 4 shows a clear increase from pre-test to post-test and that a ceiling effect is present in the posttest. Figures 5 and 6 show a shift from pre-test to post-test. For instance, the number of low-proficiency students to the left of the dotted vertical line (representing a score of less than half) decreased, and the number of medium-proficiency students (to the right of the dotted vertical line) increased. The overall level of grammatical sensitivity in the class improved.

\section{Conclusion}

The study investigated and implemented the proposed teaching procedure: combining FS with FonF. From observing the classes, students had many opportunities to accumulate FS and to notice linguistic patterns in an interactive classroom. Throughout the storytelling, dictogloss, and making a skit, the students imitated, repeated, and replaced part of the FS. The results of S-GJTs showed how much students paid attention to the patterns. The increases in their scores between the pre- and post-test S-GJTs can be summarized as follows:

1) Incorrect insertion between Vi and preposition (31\% to $59 \%$, increased by $28 \%$ )

2) Incorrect insertion between Vi and preposition (56 to $78 \%$, increased by $22 \%$ )

3) $S V$ disagreement ( $41 \%$ to $63 \%$, increased by $22 \%$ )

4) Omission -ing ( $70 \%$ to $85 \%$, increased by $15 \%$ )

5) Incorrect word order: Inverted VO (46\% to $51 \%$, increased by $5 \%)$

In summary, we observed clear improvements for all grammar items, save for inverted $V O$, which was taught using both FS and explicit grammar explanation. Regarding the grammar item inverted, this may be acoustically less salient for these students, and the word order differs from Japanese (L1). On the other hand, correctness and incorrectness (insertion, omission, and agreement) may be acoustically more salient and easier to learn through FS. For these grammar items, FS may support language use until declarative knowledge catches up and coexists with procedural knowledge.

In conclusion, FS can enhance students' grammatical sensitivity to linguistic patterns and exert a positive influence on language acquisition in terms of finding linguistic patterns and inferring meanings, as well as developing procedural knowledge, in which students learn a new language through "instance to rule." The students had not been mature enough to process the abstract rule beforehand when they encountered a particular target structure in the conceivable context. They were given opportunities to repeat and imitate what they heard and work together to reconstruct the story in dictogloss. They attempted to establish FMCs and may have noticed the part of verb -ing, analyzed the form, and made their own hypotheses. The teacher also demonstrated a grammar explanation, eliciting their hypotheses of new language properties. Afterwards, the students told the J-JTE (Y), "We understood the grammatical structure firmly in this way." All the procedures might be fundamental language experiences. However, we should also note that the procedures of FS are in ongoing use in classroom interaction, and language is learned from participatory experience of processing input and producing language during interactions in social contexts (Ellis, 2008; Larsen-Freeman, 2015), as UBM emphasized. This may explain why the present study found improvement of the whole classroom, in which both fast and slow learners moved forward. The number of medium-proficiency students increased. In terms of this outcome, students might also have gone over their collaborative tasks and shared what they had learned. In other words, FS needs to be realized in this kind of interactive classroom where students compare current consequences with antecedents they understood before.

One of the limitations of this study was that we did not compare a treatment group with a contrast group because the students are at the beginning of the introductory period of English education. Second, the research focused on the students' receptive ability (listening) in establishing FMCs. Although students' production was observed in the classroom, production through FS should be subjected to closer empirical study. Additionally, other grammar items require further study regarding the degree of the difficulty of grammar items for Japanese students.

By 2020, regulated elementary school English activities will take up 70 hours of classroom time each year, and children will start learning English at the age of 9. The findings were significant in providing data for how important it is for teachers to understand the students' procedural skills before shifting to English lessons in junior high school. Last, the procedural knowledge accessing minimum processing unit is possible to be built up on unceasingly and the process is a foundation of language learning.

\section{Declaration of Conflicting Interest}

The research was conducted gratifying the ethical standard as the participants were informed that test data is analyzed statistically and their personal information remain anonymous. The authors declare that there is no 
conflict of interest.

\section{Acknowledgements}

This paper is funded by a grant from the Japan Society for the Promotion of Science (No.17K03010), from 2017 to 2021 .

The authors would like to thank Dr Takayuki Nakamori for his valuable comments on this article. Grateful thanks also to the reviewers of English Language Teaching of the early draft of this article.

\section{References}

Anderson, J. (1993). Rules of the mind. Hillsdale, NJ: Erlbaum.

Anderson, J., Fincham, J., \& Douglass, S. (1997). The role of examples and rules in the acquisition of a cognitive skill. Journal of Experimental Psychology: Learning, Memory, and Cognition, 24(4), 932-945. http://doi.org/10.1037/0278-7393.23.4.932

Brock, A. (1986). The effects of referential questions on ESL classroom discourse. TESOL Quarterly, 20, 47-59. http://doi.org/10.2307/3586388

Bybee, J. (1995). Regular morphology and the lexicon. Language and Cognitive Process, 10, 435-455. https://doi.org/10.1080/01690969508407111

Bybee, J. (2008). Usage-based grammar and second language acquisition. In P. Robinson, \& N. C. Ellis (Eds.), Handbook of cognitive linguistics and second language acquisition (pp. 216-236). New York, NY: Routledge.

Doughty, C., \& Williams, J. (1998). Pedagogical choices in focus on form. In C. Doughty, \& J. Williams (Eds.), Focus on form in classroom second language acquisition (pp. 197-261). New York, NY: Cambridge University Press.

Dulay, H. C., \& Burt, M. K. (1974). Natural sequences in child second language acquisition. Language Learning, 24, 37-53. http://doi.org/10/111/j-1467-1770-1974.tb00234.x

Ellis, N. C. (2008). Usage-based and form-focused SLA: The implicit and explicit learning of constructions. In A. Typer, T. Kim, \& M. Takada (Eds.), Language in the context use (pp. 93-120). New York, NY: Mouton de Grater. http://doi.org/10.1515/9783/10199123

Ellis, N. C. (2012). Formulaic language and second language acquisition: Zipf and the phrasal Teddy Bear. Annual Review of Applied Linguistics, 32, 17-44. https://doi.org/10.1017/S0267190512000025

Ellis, N. C., \& Larsen-Freeman, D. (2009). Constructing a second language: Analyses and computational simulations of the emergence of linguistic constructions from usage. Language Learning, 59, 90-125. http://doi.org/10.1111/j.1467-9922.2009.00537.x

Ellis, R. (2005). Measuring implicit and explicit knowledge of a second language: A psychometric study. Studies in Second Language Acquisition, 27, 141-172. https://doi.org/10.1017/S0272263105050096

Eskildsen, S. (2012). L2 negation constructions at work. Language Learning, 62, 335-372. https://doi.org/10.1111/j.1467-9922.2012.00698.x

Gass, S. (1997). Input, interaction and the second language learner. Mahwah, NJ: Erlbaum.

Goldberg, A. (1995). Construntions: A constructions grammar approach to Argument structure. Chicago, IL: University of Chicago Press.

Hunt, R. (2003). Castle adventure in Oxford reading tree. Oxford University Press.

Izumi, E., \& Isahara, H. (2004). Investigation into language learners' acquisition order based on an error analysis of a learner corpus. IWLeL 2004: Ab Interactive Workshop on Language e-Learning, 63-71.

Izumi, S. (2009). New English education introducing focus on form approach. Tokyo: Taisyukan.

Kairyudo. (2014). Sunshine English course 1. Tokyo: Kairyudo.

Kashiwag, K. (2012). Children's form-meaning connections to verb phrases and exemplar-based learning in Japanese elementary school. ALELE, 23, 13-24.

Kidd, E., Lieven, E., \& Tomasello, M. (2010). Lexical frequency and exemplar-based learning effects in language acquisition: Evidence from sentential complements. Language Science, 32(1), 132-142. https://doi.org/10.1016/j.langsci.2009.05.002 
Langacker, R. (2000). A dynamic usage-based model. In M. Barlow, \& S. Kemmerer (Eds.), Usage-based models of language (pp. 1-63). Stanford, CA: CSLI Publications.

Larsen-Freeman, D. (2015). Research into practice: Grammar learning and teaching, Language Teaching, 41, 263-280. http//doi.org/10.1017/S0261444814000408

Logan, G. (1988). Towards an instance theory of automatization. Psychological Review, 95(4), 492-527. https://doi.org/10.1037/0033-295X.95.4.492

Long, M. (1997). Focus on form in task-based language teaching. Retrieved from http://woucentral.weebly.com

Long, M., \& Robinson, P. (1998). Focus on form: Theory, research and practice. In C. Doughty, \& J. Williams (Eds.), Focus on form in classroom second language acquisition (pp. 15-41). New York, NY: Cambridge University Press.

Loewen, S. (2009). Grammaticality judgement tests and measurement of implicit and explicit L2 knowledge. In R. Ellis, S. Loewen, C. Eleder, R. Erlam, J. Philp, \& H. Renders. (Eds.), Implicit and explicit knowledge in second language learning, testing and teaching (pp. 94-112). Bristol, UK: Multilingual Matters.

Menyuk, P., \& Brisk, M. (2005). Language development and education: Children with varying language experience. New York, NY: Palgrave MacMillan. https://doi.org/10.1057/9780230504325

MEXT. (2008). Gakusyu Sidou Youryou Kaisetsu Shougakkou Gaikokugo Katsudou Hen [Commentary on elementary school curriculum guideline for elementary foreign language activities in Japan]. Retrieved from http://www.mext.go.jp/a menu/shotou/gaikokugo/kanren/index.htm

MEXT. (2017). Action plan for the innovation of English education in Japan suitable for globalized society. Retrieved from http://www.mext.go.jp/a_menu /_icsFiles/afieldfile//2014/01/31/1343704_01.pdf

Muranoi, H. (2006). SLA research and second language learning and teaching. Tokyo: Taisyukan.

Myles, F. (2004). From data to theory: The over-representation of linguistic knowledge in SLA. Transactions of the Philological Society, 102(2), 139-168. http://doi.10.1111/j.0079-1636.2004.00133.x

Myles, F., \& Cordier, C. (2017). Formulaic sequence (FS) cannot be an umbrella term in SLA: Focusing on psycholinguistic FSs and their identification. Studies in Second Language Acquisition, 39, 3-28. http://doi.10.1017/S027226311600036X

Nakamori, T. (2009). Chunking and instruction. Tokyo: Hituzi Shobo Publishing.

Sato, R. (2011). Reconsidering the effectiveness and suitability of PPP and TBLT in the Japanese EFL classroom. JALT Journal, 32(2), 189-200.

Schmidt, R. (1995). Consciousness and foreign language learning: A tutorial on the role of attention an awareness in leaning. In R. Schmidt (Ed.), Attention and awareness in foreign language learning (pp. 1-63). Honolulu, HI: University of Hawaii Press.

Shimada, K. (2014). Grammaticality and judgement and certainty. Chubu Journal, 43, 293-298.

Shintani, N. (2012). A comparative study of the effects of input-based and production-based instruction on vocabulary acquisition by young EFL leaners. Language Teaching Research, 15(2), 137-158. https://doi.org/10.1177/1362168810388692

Shintani, N. (2013). The effect of Focus on Form and Focus on Forms Instruction on the acquisition productive knowledge of L2 vocabulary by young beginning - level learners. TESOL quarterly, 47(1), 36-62. http://doi.org/10.1002/tesq.54

Shirahata, T. (1988). The learning order of English grammatical morphemes by Japanese high school students. JACET Bulletin, 19, 83-102.

Shirahata, T., Suda, K., \& Wakabayashi, S. (2006). Eigo shutoku no jyoshiki hijoshiki dainigengo shutoku kara no kensho [Rationality or Irrationality of English language learning: An investigation from the view of second language acquisition]. Tokyo: Taisyukan.

Skehan, P. (1998). A cognitive approach to language learning. Oxford, UK: Oxford University Press.

Tomasello, M. (1992). First verbs: A case study of early grammatical development. New York, NY: Cambridge University Press. https://doi.org/10.1017/CBO9780511527678

Tomasello, M. (1998). The new psychology of language: Cognitive and functional approaches to language structure. London, UK: Lawrence Erlbaum Associates. 
Tomasello, M. (2003). Constructing a language: A usage-based theory of language acquisition. Cambridge, MA: Harvard University Press.

Urata, T., Kashiwagi, K., \& Nakata. (2014). Komyunikeisyon nouryoku no soji karkisoheto musubu syouchurenkei rinku yunitto no souzou: jirei gakusyu to kisoku gakusyu no tsunagari wo toshite [Creating units of better linkage between elementary school and junior high school on English education: Focusing on the relation between item-based learning and rule-based learning]. JES Journal, 14, $244-259$.

VanPatten, B., \& Cadierno, T. (1993). Explicit instruction and input processing. Studies in Second Language Acquisition, 15(2), 225-243. http://doi.org/10.1017/s0272263100011979

VanPatten, B., Williams, J., \& Rott, S. (2004). Form-meaning connections in second language acquisition. In B. VanPatten, J. Williams, S. Rott, \& M. Overstreet (Eds.), Form-meaning connections in second language acquisition (pp. 1-26). Mahwah, NJ: Lawrence Erlbaum Associates.

Wajnryb, R. (1990). Grammar dictation. Oxford, UK: Oxford University Press.

Xavier, G. (2013). The construct validity of grammaticality judgement tests as measures of implicit and explicit knowledge. Studies in Second Language, 35(3), 423-449. http://doi.org/10.1017/S0272263113000041

Yamaoka, T. (2005). From item-learning to category-learning: A learning process of procedural knowledge of language. ARELE, 16, 21-30.

Yamaoka, T. (2006). On the importance of imitation and repetition in foreign language learning. ARELE, 17, $1-10$.

Yamaoka, T. (2008). Syougakkou eigogakusyuu ni okeru ninchiteki sokumen: Ninchiteki hattatsu dankai ni sokushita gakusyuu to sono sokushin [On the cognitive aspects of English learning in elementary school: Efficient learning of English in congruence with cognitive development stages of the students]. Kyouiku Jissengaku Ronsyuu, 9, 75-86.

\section{Notes}

1. Usage-based model (UBM) is emphasized as follows: The central processing tenet is that language structure emerges from language use (Langacker, 2000; Bybee, 1995; Tomasello, 1998). Tomasello (2003) stated that the grammatical dimension of language is a product of a set of historical and genetic processes to collectively as grammaticalization. That is, the language patterns of use emerge and become consolidated into grammatical constructions.

2. FMCs; A cognitive operation the learners imitate and repeat what they hear and connect the meaning with English sounds, which is called "exemplar-based learning." It is defined as form-meaning connections (FMCs; Skehan, 1998, p. 54).

3. C-units: The quality of speech production is measured by the number of words per C-unit (an utterance, phrase, or sentence) that gives referential or pragmatic meaning to an interaction (Brock, 1986; e.g., A: Where is my hat?; C-unit =1; B: On the table; $\mathrm{C}$-unit = 1).

4. Procedural knowledge is about how to things. The sum of procedures operating on an individual's declarative knowledge.

5. Declarative knowledge, in contrast, is concerned with the structure of stored knowledge and language users' underlying knowledge about linguistic structure.

6. Dual mode, in which the learners' procedural knowledge and declarative knowledge coexist in language learning, is defined by Skehan (1998).

7. Token frequency is the frequency of particular items and encompasses the comprehension and use of concrete pieces of language-item and phrases (collocation) (Bybee, 1995). Token frequency strengthens memory representations for linguistic forms and makes it more accessible. It supports the learners' autonomy.

8. Type frequency is the frequency that different actual forms occur in the same language slot. Type frequency leads to categorization and analogy-forming of input and is a major factor for determining the degree of productivity of constructions (Bybee, 1995).

9. Dictogloss is an approach of grammar dictation (Wajnryb, 1990). The teacher reads a story, and the students work in pairs and try to reconstruct what they just heard. The focus is on both meaning and form to reconstruct the story with four skills in collaborative work. 
10. Structured input (VanPatten \& Cadierno, 1993) is where the students are immersed in abundant input provided by the teacher and where the students then attempt to establish FMCs. The input needs to involve target structures corresponding to the context. If the students develop input processing and intake, the target structures or linguistic patterns may be noticed by the learners while they hearing these patterns and structures.

\section{Appendix 1}

The scripts of Exemplars in Storytelling (Kairyudo, 2014)

New Year's Visit

Page1

Mother: Can anyone answer the phone? I can't answer it now.

Daughter: I'm sorry, I can't. I'm changing my clothes.

Mother: Sam?

Son: Sorry, mom. I'm drying my hair.

Mother: Jim, can you answer the phone?

Father: Sorry, I can't. Aiko. I'm brushing my teeth.

Mother: Oh, no!

\section{Page 2}

Son: Mom! It's Grandma. What are you doing?

Mother: I'm putting on my kimono. Take a message, please.

Son: Mom, Grandpa is making ozoni for us. It's almost ready.

Mother: Oh, then we can't be late.

Father: No, we can't. Let's hurry.

Daughter: I like his ozoni. It's very good.

\section{Appendix2}

\section{The scripts of exemplars in Text Book}

\section{Dialogue 1}

Can you help me? / Sorry, I can't. I'm cooking now.

\section{Dialogue 2}

Are you studying now? / No, I'm not. I'm listening to music.

\section{Dialogue 3}

What are you doing? / I'm watching TV.

\section{Copyrights}

Copyright for this article is retained by the author(s), with first publication rights granted to the journal.

This is an open-access article distributed under the terms and conditions of the Creative Commons Attribution license (http://creativecommons.org/licenses/by/4.0/). 